\title{
ECS-Komplex - ein neuer Biomarker bei Wachstumshormonstörungen?
}

\author{
Wilhelm Gossing *, Lars Radke, Marcus Frohme, Henrik Biering
}

\section{Zusammenfassung}

Störungen im Metabolismus des Wachstumshormons können sich als Wachstumshormonmangel oder -überschuss (Akromegalie) äußern. In beiden Fällen handelt es sich um seltene Krankheiten, die aufwendig behandelt werden müssen, da jeder Patient anders auf seine jeweilige Medikation anspricht. Zur Bestimmung des individuellen Ansprechverhaltens auf die medikamentöse Behandlung wird Insulin-like Growth Factor 1 verwendet, das jedoch als unzuverlässig für die Therapiekontrolle gilt. Um die Diagnostik und Therapie von Wachstumshormonstörungen zu verbessern, wurde in dieser Pilotstudie der Nutzen des neuen potenziellen Biomarkers Elongin B/CCullin5-Socs-box Komplex (ECS-Komplex) überprüft. Die fünf Proteine des Komplexes üben zusammen einen negativen Feedback-Mechanismus auf den Wachstumshormonrezeptor aus und regulieren ihn in Abhängigkeit vom Wachstumshormonspiegel im Blut. Für diese Pilotstudie wurden vier Patienten mit Wachstumshormondefizienz und 15 Patienten mit Akromegalie rekrutiert. Die Messung von Unterschieden in der Expression der Gene auf RNAEbene in Blutproben der Patienten erlaubt erste Aussagen über deren Eignung als therapeutische Marker für diese Krankheiten.

\begin{abstract}
Growth hormone (GH) dysfunctions can occur as a $\mathrm{GH}$-deficiency (GHD) or an overproduction of $\mathrm{GH}$, leading to acromegaly. Both are rare diseases, which have to be treated for many years before the correct individual dosage is found and a mitigation of symptoms can be achieved. Current medical therapy is determined by the levels of the insulin-like growth factor-1, which is considered to be an unreliable theranostic tool. In order to improve the diagnosis and therapy of patients with GH-dysfunctions, we investigated the benefits of the novel potential biomarker Elongin B/C-Cullin5-Socs-box complex (ECS-complex). Together, these proteins regulate the growth hormone receptor levels according to the blood $\mathrm{GH}$ concentration through a negative feedback loop. For this study, we were able to recruit four patients with a GHD and 15 patients with acromegaly.
\end{abstract}

The detected differential expression of the ECS-complex in patients with growth hormone dysfunctions allows for first conclusions about the potential of those proteins as predictive biomarker molecules for individualized therapies.

\section{Einleitung}

Das Wachstumshormon (GH) wird als Peptidhorm von der Hypophyse ausgeschüttet und ist hauptsächlich für das Zell- und Knochenwachstum zuständig (Ahmed \& Farquharson 2010). Darüber hinaus reguliert $\mathrm{GH}$ den Energie- und Fettstoffwechsel, die Muskelmasse, die Sexual- und weitere Funktionen (Grumbach et al. 1998).

Diese Arbeit beschäftigt sich mit zwei Stoffwechselkrankheiten, die durch Störungen des Wachstumshormonmetabolismus verursacht werden: Die Wachstumshormondefizienz (GHD) äußert sich u.a. durch eine verringerte GH-Produktion in der Hypophyse, während die Akromegalie durch eine Überproduktion entsteht. Beide Krankheiten führen zu kardiovaskulären Beeinträchtigungen und dadurch zu einer erhöhten Mortalität. Zudem haben die Stoffwechselstörungen erhebliche Auswirkungen auf körperliche Leistungsfähigkeit und äußeres Erscheinungsbild, wodurch die Lebensqualität der Patienten verringert wird (Reed et al. 2013).

Häufigster Auslöser der GHD sind genetische Prädispositionen, wobei die genaue Ursache oftmals unklar bleibt.
Krankheitsauslösende Mutationen können beispielsweise die $\mathrm{GH}$-sezernierenden Zellen der Hypophyse, die für das $\mathrm{GH}$ codierenden Gene oder etwa andere Proteine der $\mathrm{GH}$-Signalkaskade betreffen (Mullis 2010). Akromegalie wird hingegen hauptsächlich durch gutartige Hypophysenadenome induziert, die am häufigsten um das 40. Lebensjahr herum auftreten. Die GHD hat eine jährliche Inzidenz von 33 Personen pro eine Million Einwohner (Stochholm et al. 2006), wohingegen Akromegalie nur bei 3-4 Personen pro eine Million Einwohner im Jahr auftritt (Holdaway \& Raja- 
soorya 1999). Die geringe Stabilität des GH im Blut erlaubt üblicherweise nicht dessen direkte Messung zum Nachweis einer GHD; stattdessen wird meist ein Provokativtest in der Form eines Insulin-Toleranz-Tests durchgeführt (Aimaretti et al. 1998).

Hauptsächlich wird die Bestimmung des insulinähnlichen Wachstumsfaktors 1 (IGF-1) im Patientenblut unterstützend zusammen mit alters- und geschlechtsabhängigen Referenzwerten für die Diagnose von GHD und Akromegalie herangezogen.

Zur Behandlung einer GHD muss das im Körper fehlende Wachstumshormon täglich durch die Gabe von rekombinant hergestelltem $\mathrm{GH}$ ausgeglichen werden.

Bei der Akromegalie sind Hirntumore die Hauptursache, sodass das schädigende Gewebe operativ entfernt bzw. durch Bestrahlung behandelt wird, um eine Tumorregression zu erreichen.

Dennoch wird häufig auch nach dem operativen Eingriff eine erhöhte $\mathrm{GH}$ Sekretion bei den Patienten festgestellt, sodass eine weiterführende Behandlung mit Medikamenten erforderlich wird. Derzeit werden zur Behandlung der Akromegalie hauptsächlich synthetische Analoga des Somatostatins verwendet, die die Ausschüttung des
$\mathrm{GH}$ bereits in der Hypophyse unterbinden. Momentan sind Lanreotid von Ipsen und Octreotid von Novartis die einzigen zugelassenen Medikamente für diese Form der Behandlung. Nur bei einer Unverträglichkeit oder Resistenz gegenüber den Somatostatin-Analoga wird das Pegvisomant, welches von Pfizer produziert wird, eingesetzt. Dies ist eine mutierte und pegylierte Form des nativen $\mathrm{GH}$ und wirkt am Wachstumshormonrezeptor (GHR) als kompetitiver Inhibitor (Fuh et al. 1992).

Die Messung des IGF-1-Serumspiegels wird zur Bestimmung des individuellen Ansprechverhaltens gegenüber den Medikamenten zwar häufig eingesetzt (Mukherjee \& Shalet 2009), dennoch gilt IGF-1 als sehr variabel und demnach unzuverlässig für die Diagnostik von Wachstumshormonstörungen (Carel \& Butler 2010, Kelnar 2010). Außerdem gibt es eine im Alter zunehmende erhebliche Überschneidung der gemessenen IGF-1-Konzentrationen bei Kranken und Gesunden (Hilding et al. 1999).

Aus diesen Gründen haben wir nach einem alternativen Biomarker gesucht und schließlich das Protein Suppressor of Cytokine Signaling 2 (SOCS2) identifiziert. Es wird bei einem Wachstumshormonstimulus zusammen mit IGF-1

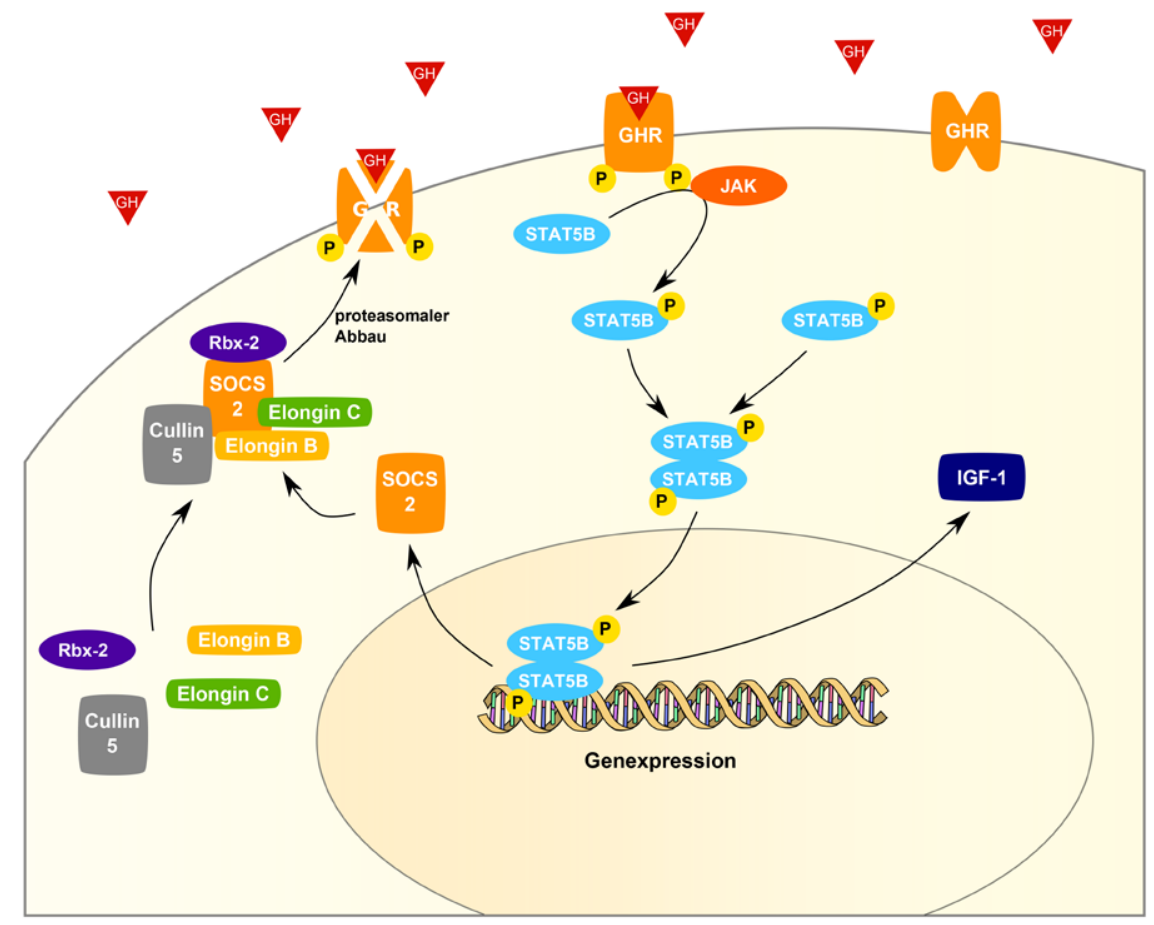

Abb. 1) Der Stoffwechselweg des Wachstumshormons induziert die Bildung des ECS-Komplexes. GH induziert die Signalkaskade am GHR, an dessen Ende STAT5B die Expression von SOCS2 induziert. Dieses tritt im Cytoplasma mit den Proteinen Elongin $B / C$, Cullin 5 und Rbx-2 zusammen und nimmt als Komplex seine regulatorische Funktion ein.

koexprimiert und reguliert den GHR in der Form eines negativen FeedbackLoops (Yang et al. 2013). Auf diese Weise fängt das SOCS2 im Körper eines gesunden Menschen exzessive $\mathrm{GH}$ Stimuli ab.

Experimente mit Knock-out-Mäusen konnten zeigen, dass SOCS2 eine tragende Rolle beim Wachstum hat (Metcalf et al. 2000). So wurden SOCS2defiziente Mäuse bis zu 40 \% größer als die Kontrollen, während sie diesen veränderten Phänotyp nicht zeigten, wenn sie gleichzeitig unter einer GHD litten (Metcalf et al. 2000).

Zudem wurde kürzlich entdeckt, dass kleinwüchsige Patienten eine höhere Expression des SOCS2-Gens haben (Ocaranza et al. 2012), wodurch letztendlich der Minderwuchs ausgelöst worden sein könnte.

Die Sensitivität der Zelle gegenüber dem GH wird hauptsächlich durch die Menge der GH-Rezeptoren in der Zellmembran reguliert. Nach der Bindung des GH wird der Rezeptor phosphoryliert und infolge einer intrazellulären Signalkaskade ubiquitinyliert, sodass dieser vom Proteasom erkannt und abgebaut wird (Leung et al. 1987) (Abb. 1). Genauer betrachtet ist ein E3-Ubiquitin-Ligase-Komplex in Verbindung mit dem SOCS2-Protein für diese Markierung mit Ubiquitin-Molekülen verantwortlich (Kamura et al. 2004).

Eine wichtige Domäne des SOCS2Proteins ist die SOCS-Box, die mit den Proteinen Elongin B, Elongin C, Cullin 5 und Ring-Box Protein 2 (Rbx2) interagiert. Zusammen bilden diese fünf Proteine den ElonginB/C-Cullin5Socs-box Complex (ECS-Complex). Dieser Komplex ist für die Funktion von SOCS2 essenziell, da er dieses stabilisiert und ebenso seinen Abbau unterbindet (Vesterlund et al. 2011). Des Weiteren sind die Elongine B und $C$ in die Regulation der Genexpression des SOCS2 involviert und beeinflussen dessen korrekte Faltung (Babon et al. 2008).

Die Inhibierung des GH-Signalwegs wird durch die Assoziation der SH2Bindedomäne des SOCS2 an die phosphorylierten Tyrosine Y487 und Y595 des GHR erreicht (Uyttendaele et al. 2007). Demnach ist SOCS2 nicht in der Lage, an inaktivierte Rezeptoren zu binden, wodurch die Zelle sensitiv gegenüber geringeren $\mathrm{GH}$-Spiegeln 
im Blut bleiben kann (Vesterlund et al. 2011). Mutationen an diesen Aminosäuren führten zu einem Funktionsverlust des SOCS2 am GHR (Greenhalgh et al. 2002). Nach der Bindung des SOCS2 an den GHR wird das Rbx2 - eine andere Komponente des ECS-Komplexes aktiv, welches nun Ubiquitin-Moleküle an den GHR anlagert, sodass dieser vom Proteasom erkannt und folglich abgebaut wird (Kamura et al. 2004). Cullin 5 ist als fünftes Protein des ECSKomplexes dafür zuständig, die Substraterkennungseinheit (SOCS2) und den enzymatischen Teil des Komplexes (Rbx2) in räumliche Nähe zueinander zu bringen (Thomas et al. 2013).

Diese Regulierung der GHR-Spiegel in der Zellmembran durch den ECSKomplex bewirkt einen negativen Feedback-Mechanismus, der die GHSensitivität der Zelle und damit das Wachstum des gesamten Organismus beeinflusst.

Demnach sind diese Proteine vielversprechende Kandidaten, um das Ansprechverhalten gegenüber zugelassenen Medikamenten bei Wachstumshormonstörungen vorauszusagen. Bei dieser Studie wurde eine Messung der Expression der fünf Gene des ECS-Komplexes in Patienten mit Wachstumshormonstörungen und gesunden Kontrollen durchgeführt, um Informationen über den potenziellen Nutzen dieses Komplexes für die individualisierte Medizin zu erlangen.

Hier stellen wir die ersten Einblicke in das transkriptionelle Verhalten des ECS-Komplexes bei Wachstumshormonstörungen vor.

\section{Material und Methoden}

\subsection{Ethikvotum und Patienten}

Neben der routinemäßigen Blutabnahme wurde eine weitere Probe für diagnostische Zwecke genommen, weswegen nach § 24 Medizinproduktegesetz ein vorheriger Ethikantrag erforderlich war. Alle Teilnehmer gaben ihr Einverständnis zur Verwendung ihres Blutes für diese Studie und zur anonymen Datenspeicherung. Ein zustimmendes Ethikvotum wurde am 04.03.2013 von der Landesärztekammer Berlin erteilt.

Vier Patienten mit einer GHD (Altersdurchschnitt 56,25 Jahre) und 15 Pa- tienten mit Akromegalie (Altersdurchschnitt 53,6 Jahre) wurden zwischen Juli und Dezember 2014 rekrutiert (Tab. 1). Einziges Einschlusskriterium war eine diagnostizierte GHD oder Akromegalie - mit oder ohne bereits bestehender Behandlung. Die Patienten mit einer GHD wurden unterteilt in neu diagnostizierte Patienten (ohne Behandlung) und Patienten mit Behandlung, die gemäß anerkannten Referenzwerten des IGF-1 außerhalb des Normalbereichs lagen. Die Akromegaliepatienten wurden unabhängig von ihrem Therapiestatus, jedoch abhängig vom angewandten Medikament in die vier Gruppen Lanreotid, Octreotid, Pegvisomant und Zustand nach erfolgreicher Hypophysenoperation unterteilt. Da das medikamentös verabreichte $\mathrm{GH}$ identisch mit dem nativen $\mathrm{GH}$ des Körpers ist, wurden vier Patienten (Durchschnittsalter 44,5 Jahre) mit IGF-1-Werten im Normalbereich (Durchschnitt IGF-1 146,5) als Kontrollgruppe in dieser Studie verwendet.

Geschlecht, Alter und Body-MassIndex (BMI) jedes Patienten wurden eingeholt, da Zusammenhänge zum Wachstumshormonhaushalt bestehen, die auf ihren Einfluss für diese Studie untersucht werden sollen. Die vorwiegend bei Frauen vorkommenden Östrogene inhibieren die intrazellulären Effekte des $\mathrm{GH}$ und haben somit auch einen möglichen Einfluss auf die SOCS2-Expression (Leung et al. 2003). Die Sekretion des $\mathrm{GH}$ verringert sich mit fortschreitendem Alter sehr stark, da die somatotrophen Zellen in der Hypophyse stetig absterben. Für die korrekte Beurteilung des Krankheitsstatus der Patienten anhand der IGF-1-Werte - und später eventuell auch anhand der Genexpression - ist daher das Alter der Patienten enorm wichtig. Darüber hinaus besteht bei Patienten mit einer Akromegalie die Gefahr einer DiabetesErkrankung, da das Wachstumshormon den Blutzuckerspiegel anhebt und gleichzeitig die Insulinresistenz erhöht (Colao et al. 2004). Deshalb wird der BMI für eine etwaige Korrelation mit der Genexpression benötigt.

\subsection{Probenvorbereitung}

Die Blutproben wurden bei den regulären vierteljährlichen Arztbesuchen der Patienten gesammelt und unver- züglich weiter bearbeitet. Durch einen Aufschluss der roten Blutkörperchen mit 0,17 M Ammoniumchloridlösung und zweifache Zentrifugation für jeweils fünf Minuten bei $453 \times \mathrm{g}$ wurden zunächst die mononukleären Zellen des peripheren Blutes (PBMCs) aus je $1,5 \mathrm{ml}$ frischen Vollblutes isoliert. Die gesamte RNA der Zellen wurde mithilfe des InviTrap Spin Universal RNA mini Kit (Stratec Molecular) extrahiert und Ertrag und Reinheit mit dem NanoDrop ND1000 (Nanodrop Instruments) gemessen. Der Quotient aus 260/280 $\mathrm{nm}$ betrug in allen Proben $>2,0$. Folgend wurde mit dem 2100 Bioanalyzer (Agilent) die Qualität kontrolliert. 300 ng RNA wurden mit Oligo(dt)-Primern und Maxima Reverser Transkriptase (Thermo Scientific) in cDNA umgeschrieben. Abschließend wurden die Proben 1:8 mit Reinstwasser (Roche Applied Science) verdünnt.

\subsection{Real-Time RT-PCR}

Die Real-Time-PCR-Ansätze enthielten 1,5 ng cDNA, $10 \mu$ l SYBR Green 1 Master (Roche Applied Science) und $5 \mu \mathrm{M}$ Forward und Reverse Primer. Jeder Ansatz wurde als Triplikat auf dem LightCycler LC480 System (Roche) wie folgt durchgeführt: 5 min Präinkubation bei $94^{\circ} \mathrm{C}$ gefolgt von 45 Amplifikationszyklen bei $94^{\circ} \mathrm{C}$ für $10 \mathrm{sec}, 61^{\circ} \mathrm{C}$ für $7 \mathrm{sec}$ und $72^{\circ} \mathrm{C}$ für $10 \mathrm{sec}$. Schmelzkurven wurden zwischen $61^{\circ} \mathrm{C}$ und $94^{\circ} \mathrm{C}$ bei einer Ramp Rate von $0,11^{\circ} \mathrm{C} / \mathrm{sec}$ erstellt. Jede 96-Well-Platte enthielt zusätzlich drei No Template Controls für jedes Primerpaar. Die Cq-Werte wurden durch die Second Derivative Maximum Method der LightCycler 480 Software 1.5.0 (Roche) berechnet. Die Experimentaldaten wurden als .txt-File exportiert und die Effizienzen der PCR mithilfe von LinRegPCR v2012.3 berechnet.

\subsection{Normalisierung und statistische Datenanalyse}

Zur Normalisierung der Genexpressionsdaten wurden die sieben Referenzgene $A C T B, B 2 M, H P R T, G A P D H$, SDHA, PPIA, YWHAZ zusätzlich in allen Proben gemessen. Die besten vier Referenzgenkandidaten konnten mithilfe der Programme geNorm, Normfinder und Bestkeeper identifi- 
Tab.1: Überblick über die Patientendaten und Einordnung in Gruppen

\begin{tabular}{|c|c|c|c|c|c|}
\hline Bezeichnung & $\begin{array}{c}\text { Therapie- } \\
\text { dauer }\end{array}$ & Geschlecht & Alter & BMI & IFG-1 \\
\hline A1 GHD unbehandelt & / & w & 57 & 35,4 & 34,6 \\
\hline A2 GHD unbehandelt & / & w & 68 & 23,0 & 17,4 \\
\hline A2 GHD unbehandelt & / & w & 65 & 28,7 & 30,3 \\
\hline C2 GHD behandelt mit IGF-1 außerhalb NB* & 6 Monate & w & 35 & 28,5 & 412,4 \\
\hline E1 Akromegalie - Lanreotid & 3 Jahre & $\mathrm{m}$ & 30 & 42,5 & 1189 \\
\hline E2 Akromegalie - Lanreotid & 3 Jahre & $\mathrm{m}$ & 60 & 38,1 & 380,4 \\
\hline E5 Akromegalie - Lanreotid & 3 Jahre & $\mathrm{m}$ & 69 & 29,7 & 152,0 \\
\hline F1 Akromegalie - Octreotid & 5 Jahre & w & 43 & 28,5 & 267,3 \\
\hline F3 Akromegalie - Octreotid & 2 Jahre & w & 46 & 37,0 & 350,5 \\
\hline G1 Akromegalie - Pegvisomant & 5 Jahre & w & 62 & 26,6 & 186,8 \\
\hline G2 Akromegalie - Pegvisomant & 7 Jahre & w & 70 & 38,1 & 207,9 \\
\hline G4 Akromegalie - Pegvisomant & 6 Jahre & $\mathrm{m}$ & 82 & 39,8 & 223,7 \\
\hline G5 Akromegalie - Pegvisomant & 4 Jahre & w & 31 & 22,0 & 168,6 \\
\hline H1 Akromegalie mit Z.n. Hypophysen-OP** & 2 Jahre & $\mathrm{m}$ & 49 & 31,8 & 91,8 \\
\hline H2 Akromegalie mit Z.n. Hypophysen-OP** & 5 Monate & $\mathrm{m}$ & 51 & 48,3 & 201,6 \\
\hline H3 Akromegalie mit Z.n. Hypophysen-OP** & 2 Jahre & w & 54 & 22,9 & 206,6 \\
\hline H4 Akromegalie mit Z.n. Hypophysen-OP** & $1 \mathrm{Jahr}$ & w & 51 & 27,8 & 170,8 \\
\hline H5 Akromegalie mit Z.n. Hypophysen-OP** & 13 Jahre & w & 51 & 32,4 & 377,4 \\
\hline H7 Akromegalie mit Z.n. Hypophysen-OP** & 15 Jahre & w & 55 & 26,0 & 163,0 \\
\hline GHD behandelt mit IGF-1 im NB (Kontrolle) & 6 Jahre & w & 43 & 30,2 & 156,9 \\
\hline GHD behandelt mit IGF-1 im NB (Kontrolle) & 2 Jahre & w & 40 & 41,9 & 147,2 \\
\hline GHD behandelt mit IGF-1 im NB (Kontrolle) & 3 Jahre & w & 59 & 20,7 & 125,6 \\
\hline GHD behandelt mit IGF-1 im NB (Kontrolle) & 7 Jahre & $\mathrm{m}$ & 36 & 21,2 & 156,2 \\
\hline
\end{tabular}

*NB $=$ Normalbereich, ** Zustand nach erfolgreicher Entfernung des Hypophysenadenoms

ziert werden. Als Ergebnis schlugen diese drei Programme übereinstimmend die Gene YWHAZ, B2M, GAPDH und PPIA als stabilste Referenzgene vor, welche daraufhin durch geometrisches Mitteln zu einem Referenzgenindex vereint wurden. Die Genexpressionswerte wurden mit der effizienzkorrigierten $\Delta \Delta$ Cq-Methode aus dem Median eines jeden Triplikats berechnet. Das statistische Signifikanzniveau wird bei dieser Studie $a b$ einem $p$-Wert von kleiner 0,05 erreicht.

\section{Ergebnisse}

Der Quotient aus 260/280 nm der RNA im Spektrometer ergab eine Reinheit von > 2,0 für alle Proben. Der mittlere RIN-Wert der RNA-Qualitätsbestimmung lag bei 9,0. Die Effizienzen der qPCR reichten von $70 \%$ bis $83 \%$ bei den Referenzgenen und von $72 \%$ bis $76 \%$ bei den Zielgenen. Des Weiteren wurde nach jedem qPCR-Lauf eine Schmelzkurvenanalyse durchgeführt, um sicherzustellen, dass ausschließlich die korrekte cDNA amplifiziert worden ist. In jeder qPCR-Reaktion entstand ein einzelner Peak an der entsprechenden Schmelztemperatur des gewünschten Produktes.

Wir hatten erwartet, in dieser Studie signifikante Genexpressionsunterschiede zwischen gesunden Menschen und Menschen mit einer diagnostizierten Wachstumshormonstörung zu finden, die mit dem IGF-1-Status korrelieren. Die Genexpression der 19 involvierten Probanden zeigte Muster, die sich mit unseren Erwartungen deckten. Während bei den Patienten mit einer GHD vier von fünf Genen des untersuchten Biomarkers signifikant reguliert wa- 
ren, zeigten sich bei den Patienten mit einer Akromegalie geringere Unterschiede. Dies steht offenbar im Zusammenhang mit den laufenden medikamentösen Behandlungen, und je nach eingesetztem Medikament ergab sich ein anderes Expressionsprofil.

Bei den unbehandelten GHD-Patienten waren die drei Gene SOCS2 $(0,54, \mathrm{p}=0,044), \operatorname{TCEB} 2(0,7, \mathrm{p}=0,05)$ und CUL5 $(0,22, p=0,002)$ signifikant herunterreguliert, während das Gen TCEB1, welches für das Elongin C COdiert, im Schnitt 6,4-fach höher als in den Kontrollen exprimiert wurde, jedoch eine hohe Standardabweichung von 3,7 aufwies $(p=0,012)$ (Abb. 2).

Da die IGF-1-Werte der untersuchten Akromegalie-Patienten durch die bereits begonnene Titration der Medikamente nahe am bzw. innerhalb des gültigen Referenzbereichs lagen, zeigten sich hier etwas geringere Unterschiede, die sich jedoch in Bezug auf die Behandlungsgruppe teilweise deutlich unterschieden (Abb. 3). Während TCEB1 und TCEB2 in der mit Lanreotid behandelten Gruppe keine ver- änderte Genexpression zeigten, waren die anderen drei Gene leicht herunterreguliert (SOCS2: 0,66, CUL5: 0,7, RNF7: $0,68)$, stellten jedoch keine signifikante Veränderung dar. Die mit Octreotid behandelten Patienten zeigten eine deutlich reduzierte Expression des CUL5-Gens (0,54, $p=0,05)$, während TCEB1 und TCEB2 erneut unverändert waren. Die mRNA-Expression von SOCS2 $(0,78)$ und RNF7 $(0,81)$ war um ca. $20 \%$ reduziert. Der GHR-Rezeptorantagonist Pegvisomant führte zu einer Zunahme der SOCS2-Transkriptmenge auf das 2,7 -Fache $(p<0,01)$ und $\mathrm{zu}$ mehr als einer Verdopplung des TCEB1-mRNA-Niveaus $(2,27, \mathrm{p}<0,001)$. Widersprüchlich ist, dass die Expression des RNF7 an dieser Stelle signifikant auf 0,5 reduziert wurde $(p=0,01)$. Die Patienten mit Zustand nach erfolgreicher Hypophysen-Operation zeigten geringe Hochregulierungen in vier der fünf Zielgene. SOCS2 (1,32), TCEB2 $(1,28)$ und RNF7 $(1,29)$ waren relativ homogen erhöht, während TCEB1 als einziges Gen signifikant erhöht vorlag $(p<0,01)$.

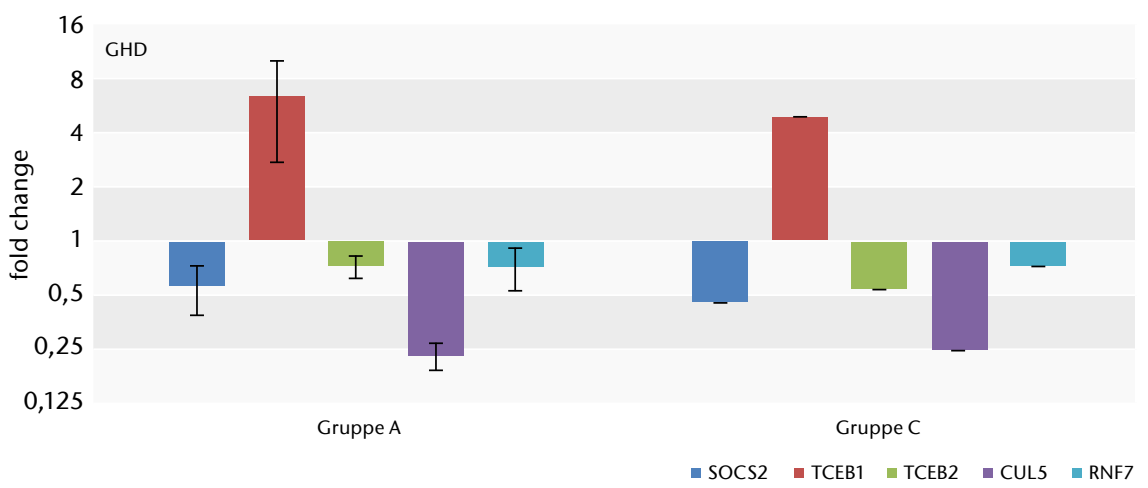

Abb. 2) Relative mRNA-Expression der fünf Gene des ECS-Komplexes der GHD-Patienten im Vergleich mit der Kontrollgruppe. Die Gruppe A umfasst drei Patienten, während in C nur ein Patient enthalten ist. Die Fehlerbalken zeigen die Standardabweichung.

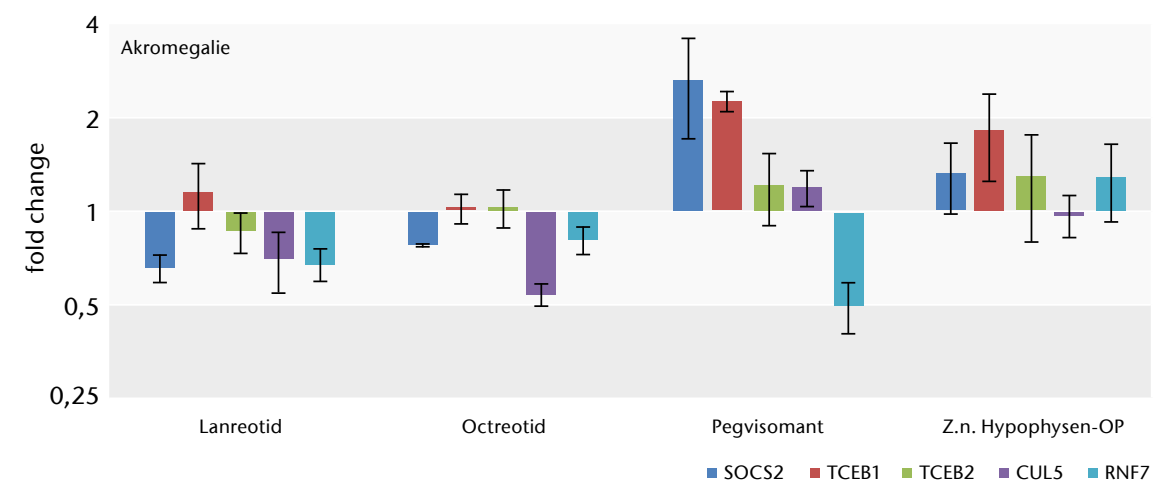

Abb. 3) Relative mRNA-Expression des ECS-Komplexes in den vier Behandlungsgruppen der Akromegaliepatienten. Die Gruppen umfassen 3, 2, 4 und 6 Patienten (von links nach rechts).

\section{Diskussion}

In dieser Pilotstudie wurden die Genexpressionsprofile von fünf Genen untersucht, die eine Schlüsselfunktion in der Regulation des GHR spielen. Es zeigte sich, dass die Gene SOCS2, TCEB2, CUL5 und RNF7 in erwachsenen Patienten mit einer unbehandelten GHD herunterreguliert waren. Es wird angenommen, dass die geringe $\mathrm{GH}$ Konzentration im Blut dieser Patienten die Aktivität des JAK-STAT-assoziierten GH-Signalwegs reduziert, weshalb die Expression der Gene des ECS-Komplexes verringert sein sollte. Außerdem ermöglichen die geringen SOCS2-Spiegel in den GHD-Patienten den Zellen, sensitiver gegenüber den ohnehin schon geringen $\mathrm{GH}-$ Konzentrationen zu sein. Die größte Abnahme der Transkriptmenge wurde im CUL5-Gen beobachtet, welches für das Cullin-5-Adapterprotein codiert, das wiederum für das Zusammenführen von Substraterkennungseinheit und Enzymteil des Komplexes verantwortlich ist.

Dasselbe Regulationsmuster wurde in dem einen GHD-Patienten beobachtet, der bereits seit sechs Monaten unter medikamentöser Behandlung stand. Dessen IGF-1-Wert von $412,4 \mathrm{ng} / \mathrm{ml}$ deutet auf eine erhöhte $\mathrm{GH}-K o n z e n t r a t i o n$ hin, jedoch zeigt die Genexpression einen verringerten Bedarf des ECS-Komplexes an. Da der IGF-1-Werte generell mehrere Wochen im Blut stabil ist, könnte dieser dem eigentlichen Status des Körpers hinterherhängen, sodass sich die eventuell niedrige $\mathrm{GH}$-Dosis nicht im IGF-1-Wert widerspiegeln würde. Dementsprechend gibt es Belege dafür, dass der GH-Status und die gemessenen IGF-1-Wert nicht immer korrelieren und sogar gegensätzliche Konzentrationen anzeigen können (Carmichael et al. 2009). Des Weiteren ist TCEB1 das einzige Gen, welches unabhängig von der Art der Wachstumshormonerkrankung bei den Patienten immer hochreguliert zu sein scheint, sodass hier eine Wechselwirkung mit einem anderen Hormon oder Regulator vermutet wird.

Für die Auswertung dieser Studie muss berücksichtigt werden, dass die Akromegalie-Patienten entsprechend ihrer Medikation in Gruppen eingeteilt wurden und nicht wie bei der GHD - 
nach Therapiestatus. Somit gibt es Patienten innerhalb und außerhalb des IGF-1-Normalbereichs in der gleichen Experimentalgruppe, wodurch die individuelle Genexpression stark vom Behandlungsstatus abhängt und die zusammengefasste Auswertung einen erhöhten Variationskoeffizienten bedingt.

Die Patienten unter Therapie mit Lanreotid zeigten eine einheitlich verringerte Genexpression in drei der fünf Gene, die auf eine übermäßige Reduktion des $\mathrm{GH}$-Spiegels durch eine geringfügige Überdosis des Somatostatin-Analogons zurückzuführen sein könnte. Obwohl diese Gruppe mit drei Proben nur eine begrenzte Aussagekraft vermittelt, deuten diese bereits eine Möglichkeit zur besseren Dosierung des Lanreotids an. Die Behandlung mit Octreotid führte wie beim Lanreotid zu keiner Veränderung von TCEB1 und TCEB2, wohingegen wiederum verringerte Transkriptspiegel der anderen drei Gene zu beobachten waren, die ebenfalls auf Effekte einer Überdosis hindeuten könnten. Zusammen betrachtet resultieren beide Somatostatin-Analoga in ähnlichen Expressionsmustern und scheinen somit denselben Einfluss auf den Metabolismus des Körpers zu haben. Die Patienten, die mit dem GHR-Antagonisten Pegvisomant behandelt wurden, zeigten mehr als zweifach hochregulierte SOCS2- und TCEB1-Spiegel, während RNF7 um die Hälfte reduziert vorlag. Das Pegvisomant senkt nicht die Menge an GH im Körper, sondern reduziert die $\mathrm{GH}$-induzierte Aktivierung des GHR durch Inhibierung seiner Dimerisation. Interessanterweise beinhaltet diese gegensätzliche Regulation die beiden enzymatischen Komponenten des Proteinkomplexes: SOCS2 und RNF7. Dies suggeriert eine Änderung des Verhältnisses der fünf Komponenten des ECS-Komplexes zueinander, was für dessen Selbstregulation eine Rolle spielen könnte. Allerdings bleibt unklar, inwiefern dieses Regulationsmuster einem bestimmten biochemischen Prozess zugeordnet werden kann, zweifellos deutet es jedoch auf einen bislang unbekannten Effekt des Pegvisomant hin.

Die Gruppe der Patienten mit Zustand nach erfolgreicher HypophysenOperation wies geringfügig erhöhte
SOCS2-, TCEB2- und RNF7-Spiegel, um 83 \% erhöhte TCEB1-Spiegel und CUL5Werte im Bereich der Kontrollgruppe auf. Trotz des erfolgreich entfernten schädlichen Adenoms und nahezu normalen IGF-1-Werten bestand dennoch eine erhöhte Genaktivität, die auf eventuell remanente Tumoraktivität oder Beeinträchtigungen des verbliebenen Gewebes zurückzuführen sein könnte. Diese Erkenntnisse eröffnen die Möglichkeit, solche Überbleibsel unvollständig entfernten Tumorgewebes zukünftig durch Analyse des ECSKomplexes besser detektieren zu können, jedoch würde dies weitere Daten durch ein größeres Patientenkollektiv erfordern.

Um den Nutzen des ECS-Komplexes für die Diagnose einer GHD einschätzen zu können, wurden neudiagnostizierte, unbehandelte GHD-Patienten rekrutiert. Diese drei Patienten zeigten in vier der fünf Gene signifikante Genexpressionsunterschiede. Insbesondere waren die Transkriptionslevel des CUL5 deutlich reduziert, weshalb dieses Gen zu einer Unterscheidung zwischen Gesund und GHD-Erkrankung nützlich sein könnte. Gerade bei älteren Menschen wäre dies von großer Bedeutung, da sich deren IGF-1-Werten oftmals mit denen von gesunden Personen überschneiden (Hilding et al. 1999). Allerdings sollte beachtet werden, dass die Werte von drei Patienten nicht für eine gesicherte Aussage ausreichen und mehr Patienten benötigt werden, um die mögliche physiologische Standardabweichung dieser Werte zu untersuchen. Aufgrund der sehr geringen Inzidenz der Akromegalie konnten keine neudiagnostizierten Akromegaliepatienten in der Zeitspanne der Patientenrekrutierung für diese Studie angeworben werden. Deshalb bleibt das diagnostische Potenzial des ECS-Komplexes für die Akromegalie undeutlich.

Überdies lassen sich Auffälligkeiten zwischen den verschiedenen Gruppen feststellen, die möglicherweise mit den verschiedenen Wirkprinzipien der Medikamente zusammenhängen. So war das SOCS2 bei den Akromegaliepatienten nur in den mit Somatostatin-Analoga behandelten Gruppen verringert, wohingegen es in der PegvisomantGruppe stark erhöht vorlag. Dazu war das TCEB1 in allen Gruppen unabhän- gig von Mangel- oder Überschusserkrankung deutlich erhöht. Nur die Somatostatin-Analoga vermochten es, die Expression des TCEB1 auf das Normalniveau zu bringen. Ebenso war die Gruppe mit Zustand nach erfolgreicher Tumorentfernung die einzige, in der die Expression des RNF7-Gens über dem Normalniveau lag. Diese Expressionsmuster sind bei allen Patienten der jeweiligen Behandlungsgruppen zu beobachten. Dementsprechend kann anhand der vorliegenden Daten bereits vermutet werden, dass die einzelnen Medikamente verschiedenartige Effekte im Körper hervorrufen, die sich zumindest in der Genexpression des ECS-Komplexes widerspiegeln.

Ein Abgleich der individuellen Genexpression mit dem Body-Mass-Index des Patienten ergab keinen augenscheinlichen Zusammenhang. Allerdings könnte solch ein Effekt nur bei unbehandelten Akromegalie-Patienten diskutiert werden, da durch die medikamentöse Therapie bereits auf die individuellen Unterschiede eingegangen wurde.

Des Weiteren kann bei einem Vergleich der individuellen Genexpression mit den IGF-1-Werten der Patienten (Daten nicht gezeigt) nur vereinzelt eine Korrelation festgestellt werden. Der Patient E1 aus der Lanreotid-Gruppe war der jüngste Patient, hatte den zweithöchsten BMI und besaß mit $1190 \mathrm{ng} / \mathrm{ml}$ bei Weitem den höchsten IGF-1-Wert. Dennoch zeigten nur die Werte des TCEB2 und CUL5 die höchsten Werte in dieser Probe. Insgesamt zeigten jedoch die Genexpression und die zugehörigen IGF-1-Werten keine direkte Proportionalität zueinander. Einerseits könnten die zuvor beschriebenen wirkstoffabhängigen Effekte dabei eine Rolle spielen, andererseits wurde bereits gezeigt, dass das IGF-1 ohnehin nicht immer korrekt den $\mathrm{GH}$ Status anzugeben vermag (Carmichael et al. 2009). Aus diesem Grund besitzt der ECS-Komplex auch ohne die Korrelation zum IGF-1 das Potenzial, das individuelle Ansprechverhalten gegenüber der Behandlung von Wachstumshormonstörungen aus einer neuen Perspektive zu beleuchten. Eine Verlaufsstudie mit einer höheren Patientenzahl und einer zusätzlichen Analyse der Proteinkonzentrationen 
der fünf ECS-Komplex-Komponenten könnte für weitere Untersuchungen zu diesem Biomarker von großem Nutzen sein.

\section{Schlussfolgerung}

Neunzehn Patienten mit Wachstumshormonstörungen wurden mit der Real-Time PCR auf Genexpressionsunterschiede im ECS-Komplex untersucht. Die drei Patienten mit einer schweren Wachstumshormondefizienz zeigten signifikant reduzierte mRNA-Konzentrationen in drei der fünf Zielgene, wodurch eine Unterscheidung zwischen normalem und abnormalem Wachstumshormonhaushalt möglich ist. Die Expression des CUL5-Gens stellte sich als sehr deutlich herunterreguliert heraus und hat somit Potenzial für die Diagnostik der GHD. Die Daten der behandelten Akromegaliepatienten zeigten einige signifikante Expressionsunterschiede, aufgrund der geringen Studienteilnehmerzahl ist jedoch keine sichere Interpretation möglich. Da IGF-1 in der Diagnostik von Wachstumshormonstörungen als Goldstandard gilt, müssen neue Erkenntnisse stets mit diesem Marker verglichen werden. Weil aber die beobachteten Genexpressionen nur teilweise mit den IGF-1-Werten korrelieren, können die gemessenen Unterschiede noch nicht zu Verbesserungen der individuellen Therapien genutzt werden.

Entscheidende Aspekte zukünftiger Untersuchungen des ECS-Komplexes im Kontext der Wachstumshormonstörungen sind ein größeres Patientenkollektiv, insbesondere mit neudiagnostizierten und unbehandelten Fällen von Akromegalie, die Messung des Komplexes auf Proteinebene und eine Langzeitstudie, in der Genexpression und Proteinkonzentration nach festgelegten Intervallen gemessen und mit dem jeweiligen Therapieverlauf abgeglichen werden. Auf diese Weise könnten Korrelationen zwischen dem Ansprechverhalten auf eine bestimmte Therapie und den gewonnenen Daten aufschlussreicher analysiert und zeitgleich die Sensitivitäten von IGF-1 und ECS-Komplex verglichen werden.

\section{LITERATUR}

Ahmed SF, Farquharson C (2010) The effect of GH and IGF1 on linear growth and skeletal development and their modulation by SOCS proteins. J Endocrino 206(3):249-259. doi: 10.1677/JOE-10-0045

Aimaretti G, Corneli G, Razzore P, Bellone S, Baffoni C, Arvat E, Camanni F, Ghigo E (1998) Comparison between Insulin-Induced Hypoglycemia and Growth Hormon $(\mathrm{CH})$-Releasing Hormone + Arginine as Provocamon Clin Endocrinol Metab 83(5):1615-1618. doi: 10.1210/ jcem.83.5.4837

Babon JJ, Sabo JK, Soetopo A, Yao S, Bailey MF, Zhang J, Nicola NA, Norton RS (2008) The SOCS Box Domain of SOCS3: Structure and Interaction with the ElonginBCCullin5 Ubiquitin Ligase. J Mol Biol 381(4):928-940. doi: 10.1016/j.jmb.2008.06.038

Carel J, Butler G (2010) Safety of Recombinant Human Growth Hormone. Endocr Dev 18:40-54. doi: man Growth Hormor

Carmichael JD, Bonert VS, Mirocha JM, Melmed S (2009) The Utility of Oral Glucose Tolerance Testing for Diagnosis and Assessment of Treatment Outcomes in 166 Patients with Acromegaly. I Clin Endocrinol Metab 94(2):523-527. doi: 10.1210/jc.2008-1371

Colao A, Ferone D, Marzullo P, Lombardi G (2004) Systemic Complications of Acromegaly: Epidemiology, mic Complications of Acromegaly: Epidemiology, Pathodoi: 10.1210/er.2002-0022

Fuh G, Cunningham BC, Fukunaga R, Nagata S, Goeddel DV, Wells JA (1992) Rational Design of Potent Antagonists to the Human Growth Hormone Receptor. Science 256:1677-1680. doi: 10.1126/science.256.5064.1677

Greenhalgh $\mathrm{Cl}$, Metcalf D, Thaus AL, Corbin JE, Uren R, Morgan PO, Fabri LI, Zhang I, Martin HM, Willson TA Billestrup N, Nicola NA, Baca M, Alexander WS, Hilton D (2002) Biological Evidence That SOCS-2 Can Act Either (2002) Biological Evidence That SOCS-2 Can Act Either as an Enhancer or Suppressor of Growth Hormone Sig-
naling. I Biol Chem 277(43):40181-40184. doi: 10.1074/ naling. J Biol Che

Grumbach MM, Bin-Abbas BS, Kaplan SL (1998) The Growth Hormone Cascade: Progress and Long-Term Results of Growth Hormone Treatment in Growth Hormone Deficiency. Horm Res 49(Suppl 2):41-57. doi: 10.1159/000053087

Hilding A, Hall K, Wivall-Helleryd IL, Sääf M, Melin AL, Thorén M (1999) Serum Levels of Insulin-Like Growth Factor I in 152 Patients with Growth Hormone Deficiency, Aged 19-82 Years, in Relation to Those in Healthy Subjects. J Clin Endocrinol Metab 84(6):2013-2019. doi 10.1210/jcem.84.6.5793

Holdaway IM, Rajasoorya C (1999) Epidemiology of Acromegaly. Pituitary 2(1):29-41. doi 10.1023/A:1009965803750

Kamura T, Maenaka K, Kotoshiba S, Matsumoto M, Kohda D, Conaway RC, Conaway JW, Nakayama KI (2004) VHL-box and SOCS-box domains determine binding specificity for Cul2-Rbx1 and Cul5-Rbx2 modules of ubiquitin ligases. Genes Dev 18(24):3055-3065. doi: 10.1101/ gad.1252404

Kelnar CJH (2010) The Evidence Base for Growth Hormone Effectiveness in Children. Endocr Dev 18:23-39. doi: ne Effectiveness in

Leung DW, Spencer SA, Cachianes G, Hammonds RG, Collins C, Henzel WJ, Barnard R, Waters MJ, Wood WI (1987) Growth hormone receptor and serum binding protein: purification, cloning and expression. Nature 330:537-543. doi: 10.1038/330537a0

Leung KC, Doyle N, Ballesteros M, Sjogren K, Watts CKW, Low TH, Leong GM, Ross RJM, Ho KKY (2003) Estrogen inhibits $\mathrm{GH}$ signaling by suppressing $\mathrm{GH}$-induced JAK2 inhibits GH signaling by suppressing GH-induced JAK2 phosphorylation, an effect mediated by SOCS-2. Proc
Natl Acad Sci USA 100(3):1016-1021. doi: 10.1073/ Natl Acad Sci USA
pnas. 0337600100

Metcalf D, Greenhalgh CJ, Viney E, Willson TA, Starr R, Nicola NA, Hilton DJ, Alexander WS (2000) Gigantism in mice lacking suppressor of cytokine signalling-2. Nature 405:1069-1073. doi: 10.1038/35016611

Mukherjee A, Shalet SM (2009) The value of IGF1 estimation in adults with GH deficiency. Eur | Endocrinol 161(Suppl 1):S33-S39. doi: 10.1530/EJE-09-0247
Mullis PE (2010) Genetics of Isolated Growth Hormone Deficiency. J Clin Res Pediatr Endocrinol 2(2):52-62. doi: $10.4274 /$ jcrpe.v2i2.52

Ocaranza P, Morales F, Román R, Iñiguez $\mathrm{G}$, Cassorla F (2012) Expression of SOCS1, SOCS2, and SOCS3 in growth hormone-stimulated skin fibroblasts from children with idiopathic short stature. I Pediatr Endocr Met 25(3-4). doi: 10.1515/jpem-2011-0491

Reed ML, Merriam GR, Kargi AY (2013) Adult Growth Hormone Deficiency - Benefits, Side Effects, and Risks of Growth Hormone Replacement. Front Endocrinol 4:64 doi: $10.3389 /$ fendo.2013.00064

Stochholm K, Gravholt CH, Laursen T, Jørgensen JO, Laurberg $\mathrm{P}$, Andersen $\mathrm{M}$, Kristensen LØ, Feldt-Rasmussen U, Christiansen IS, Frydenberg M, Green A (2006) Incidence of $\mathrm{CH}$ deficiency - a nationwide study. Eur I Endocrinol 155(1):61-71. doi: 10.1530/eje.1.02191

Thomas JC, Matak-Vinkovic D, van Molle I, Ciulli A (2013) Multimeric Complexes among Ankyrin-Repeat and SOCS-box Protein 9 (ASB9), ElonginBC, and Cullin 5: Insights into the Structure and Assembly of ECStype Cullin-RING E3 Ubiquitin Ligases. Biochemistry 52(31):5236-5246. doi: 10.1021/bi400758h

Uyttendaele I, Lemmens I, Verhee A, Smet A de, Vandekerckhove I, Lavens D, Peelman F, Tavernier I (2007) dekerchove J, Lavens D, Peelman F, Tavernier J (2007) PIT) Analysis of STAT5, CIS, and SOCS2 Interactions with the Growth Hormone Receptor. Mol Endocrino with the Growth Hormone Receptor. Mol Endo

Vesterlund M, Zadjali F, Persson T, Nielsen ML, Kessle BM, Norstedt G, Flores-Morales A (2011) The SOCS2 Ubiquitin Ligase Complex Regulates Growth Hormone Receptor Levels. PLoS ONE 6(9):e25358. doi: 10.1371/ journal.pone.0025358

Yang HL, Feng M, Tan X, Yan GY, Sun C (2013) The role of SOCS2 in recombinant human growth hormone $(\mathrm{rhGH})$ regulating lipid metabolism in high-fat-diet-induced obesity mice. Mol Biol Rep 40(3):2319-2326. doi: 10.1007/ s11033-012-2313-5

Abbildungsverzeichnis

Abb. 1: GH-induzierter negativer Feedback-Mechanismus des S-Komplexes

Abb. 2: Relative Genexpression des S-Komplexes bei Patienten mit GHD

Abb. 3: Relative Genexpression des S-Komplexes bei Patienten mit Akromegalie

\section{AUTOREN}

Wilhelm Gossing, M. Sc

Lars Radke, M. Sc.

Marcus Frohme, Prof. Dr. sc. hum.

Technische Hochschule Wildau

Forschungsgruppe Molekulare Biotechnologie und funktionelle Genomik

Henrik Biering, Dr. med

Internist, Endokrinologie/Diabetologie

Praxis an der Kaisereiche, Berlin

E-Mail für Korrespondenz:

wilhelm.gossing@th-wildau.de 